\title{
On the observability of T Tauri accretion shocks in the X-ray band
}

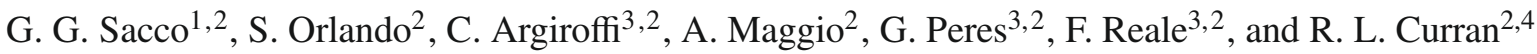 \\ 1 Chester F. Carlson Center for Imaging Science, Rochester Institute of Technology, 54 Lomb Memorial Dr., Rochester, NY 14623 , \\ USA \\ e-mail: sacco@cis.rit.edu \\ 2 INAF - Osservatorio Astronomico di Palermo, Piazza del Parlamento, 1, 90134 Palermo, Italy \\ 3 DSFA - Università degli Studi di Palermo, Piazza del Parlamento, 1, 90134 Palermo, Italy \\ 4 Department of Physics, Rochester Institute of Technology, 54 Lomb Memorial Dr., Rochester, NY 14623, USA
}

Received 6 May 2010 / Accepted 13 July 2010

\section{ABSTRACT}

\begin{abstract}
Context. High resolution X-ray observations of classical T Tauri stars (CTTSs) show a soft X-ray excess due to high density plasma $\left(n_{\mathrm{e}}=10^{11}-10^{13} \mathrm{~cm}^{-3}\right)$. This emission has been attributed to shock-heated accreting material impacting onto the stellar surface.

Aims. We investigate the observability of the shock-heated accreting material in the X-ray band as a function of the accretion stream properties (velocity, density, and metal abundance) in the case of plasma- $\beta \ll 1$ (thermal pressure « magnetic pressure) in the postshock zone.

Methods. We use a 1-D hydrodynamic model describing the impact of an accretion stream onto the chromosphere of a CTTS, including the effects of radiative cooling, gravity stratification and thermal conduction. We explore the space of relevant parameters and synthesize from the model results the X-ray emission in the [0.5-8.0] keV band and in the resonance lines of O VII (21.60 A) and Ne IX (13.45 ̊), taking into account the absorption from the chromosphere.

Results. The accretion stream properties largely influence the temperature and the stand-off height of the shocked slab and its sinking in the chromosphere, determining the observability of the shocked plasma affected by chromospheric absorption. Our model predicts that X-ray observations preferentially detect emission from low density and high velocity shocked accretion streams due to the large absorption of dense post-shock plasma. In all the cases examined, the post-shock zone exhibits quasi-periodic oscillations due to thermal instabilities with periods ranging from $3 \times 10^{-2}$ to $4 \times 10^{3} \mathrm{~s}$. In the case of inhomogeneous streams and $\beta \ll 1$, the shock oscillations are hardly detectable.

Conclusions. We suggest that, if accretion streams are inhomogeneous, the selection effect introduced by the absorption on observable plasma components may easily explain the discrepancy between the accretion rate measured by optical and X-ray data as well as the different densities measured using different He-like triplets in the X-ray band.
\end{abstract}

Key words. accretion, accretion disks - hydrodynamics - shock waves - stars: pre-main sequence - X-rays: stars

\section{Introduction}

Young accreting stars, also called classical $\mathrm{T}$ Tauri stars (CTTSs), have been well studied during the last decades, both from the observational and theoretical point of view. On the basis of the largely accepted magnetospheric accretion scenario, gas from the circumstellar disk accretes onto the star surface following the magnetic field lines of the stellar magnetosphere (Koenigl 1991). Since the circumstellar disk is truncated at $\sim 3-10$ stellar radii, accreting gas is accelerated up to velocities of $\sim 200-600 \mathrm{~km} \mathrm{~s}^{-1}$ by the stellar gravitational field and, when impacting on the high-density layers of the stellar atmosphere, it forms a strong shock that heats up the accretion column to temperatures of few MK (Gullbring 1994; Lamzin 1998). The magnetospheric accretion paradigm is supported by various observational evidence: optical emission lines with inverse P Cygni profiles indicate infalling material (e.g. Edwards et al. 1994), UV and optical excess comes from a photosphere heated by the accretion shock (Calvet \& Gullbring 1998; Gullbring et al. 2000), and transition emission lines in the far-UV are produced by the shock-heated material at temperature of about $10^{5} \mathrm{~K}$ (e.g. Ardila et al. 2002; Herczeg et al. 2005).

More recently, high resolution $(R \sim 600)$ X-ray observations of some CTTSs (TW Hya, BP Tau, V4046 Sgr, Hen 3-600,
MP Mus and RU Lupi) have revealed a soft X-ray excess produced by plasma at temperature $T \sim 2-3 \times 10^{6} \mathrm{~K}$ and electron number density $n_{\mathrm{e}} \sim 10^{11}-10^{13} \mathrm{~cm}^{-3}$ (Kastner et al. 2002; Schmitt et al. 2005; Günther et al. 2006; Huenemoerder et al. 2007; Argiroffi et al. 2007; Robrade \& Schmitt 2007). This component could be produced by the shock-heated plasma at the base of the accretion column, the plasma density being much higher than that $\left(n_{\mathrm{e}} \leq 10^{10} \mathrm{~cm}^{-3}\right)$ measured for coronae of active stars (Testa et al. 2004). The discovery of optical depths effects on the X-ray spectrum of MP Mus further supports this hypothesis (Argiroffi et al. 2009).

The idea that the soft X-ray excess detected in CTTSs is due to shocks formed at the impact of accretion columns onto the stellar surface has recently received a convincing theoretical support by time-dependent models of radiative accretion shocks in CTTSs (Koldoba et al. 2008; Sacco et al. 2008; Orlando et al. 2010). In particular, these studies have predicted post-shock plasma characterized by density and temperature values in the range observed and global shock oscillations induced by radiative cooling instabilities analogous to those predicted in other astrophysical contexts (e.g. Sutherland \& Dopita 1993; Dopita \& Sutherland 1996; Safier 1998; Sutherland et al. 2003a,b; Mignone 2005). Sacco et al. (2008; hereafter Paper I) developed 
a one-dimensional hydrodynamic model with the aim to investigate the dynamics and the stability of the shock-heated accreting material and the role of the stellar chromosphere in determining the position and the thickness of the shocked region. The model takes into account all the important physical effects, i.e the gravity stratification, the thermal conduction, the radiative losses from an optically thin plasma, and a detailed description of the stellar chromosphere. For simulations based on the parameters of MP Mus, they synthesized the high resolution X-ray spectrum, as it would be observed with the Reflection Grating Spectrometers (RGS) on board the XMM-Newton satellite, and found an excellent agreement with the observations (Argiroffi et al. 2007).

Although the time-dependent models of radiative shocks support well the origin of the soft X-ray excess in CTTSs, several observational points remain still unclear: (a) the mass accretion rates derived from X-ray observations are generally lower than those derived from optical and UV data by one or two orders of magnitude (Schmitt et al. 2005; Günther et al. 2007; Argiroffi et al. 2009; Curran et al. 2010); (b) some young accreting objects (T Tau, AB Aur, HD 163296) do not exhibit spectral features indicating dense X-ray emitting plasma (Güdel et al. 2007; Telleschi et al. 2007; Günther \& Schmitt 2009); (c) a detailed time series analysis of the soft X-ray emission from TW Hydrae revealed no periodic variations (Drake et al. 2009). In addition, the absorption from the stellar atmosphere could play an important role in the observability of accretion shocks in X-rays, especially for high density streams (Drake 2005), but up to now this effect has been not fully explored.

More recently, Brickhouse et al. (2010) performed a long exposure X-ray observation of TW Hydrae, using the Chandra High Energy Trasmission Grating, that provides a rich set of diagnostics for electron temperature, electron density and the hydrogen column density. Temperature $\left(T_{\mathrm{e}} \approx 2.5 \mathrm{MK}\right)$ and electron density $\left(n_{\mathrm{e}} \approx 3.0 \times 10^{12} \mathrm{~cm}^{-3}\right)$ derived from the He-like Ne IX line ratio diagnostics are in agreement with a standard accretion shock model describing a single stream with uniform density and velocity. However, this model cannot explain the lower values of plasma density $\left(n_{\mathrm{e}} \approx 6.0 \times 10^{11} \mathrm{~cm}^{-3}\right)$ and temperature $\left(T_{\mathrm{e}} \approx 1.5 \mathrm{MK}\right)$ derived from the $\mathrm{O}$ VII line ratio, because single stream models predict that the density of the post-shock plasma increases as the temperature decreases. In order to explain both the O VII and Ne IX diagnostics, Brickhouse et al. (2010) suggested that the X-ray emission originates from three plasma components: a hot $\left(T_{\mathrm{e}} \approx 10 \mathrm{MK}\right)$ corona, a high density $\left(n_{\mathrm{e}} \approx 6.0 \times 10^{12} \mathrm{~cm}^{-3}, T_{\mathrm{e}} \approx 3.0 \mathrm{MK}\right)$ post-shock region close to the shock surface, and a cold less dense $\left(n_{\mathrm{e}} \approx 2 \times 10^{11} \mathrm{~cm}^{-3}\right.$, $T_{\mathrm{e}} \approx 2.0 \mathrm{MK}$ ) post-shock cooling region, with 300 times more volume and 30 times more mass than that of the post shock region itself.

In this paper we analyze the observability of the X-ray emission from the post-shock plasma as a function of the properties of the accretion stream (density, velocity, and metal abundance). The main targets of our work are: (a) determining the main signatures of the accretion shock in the X-ray band; (b) estimating how the X-ray luminosity from the shock-heated plasma depends on the properties of the accretion stream; (c) investigating the influence of the absorption from the stellar atmosphere on the observed X-ray emission from the post-shock zone; (d) understanding if the discrepancy between accretion rates measured from optical and X-ray data as well as the different densities measured from the O VII and Ne IX He-like ions can be explained in the framework of 1-D hydrodynamic models, assuming plasma- $\beta \ll 1$. To answer these questions, we perform a set of 1-D hydrodynamical simulations using the model introduced in Paper I. We explore the parameter space of the accretion stream, namely its mass density, velocity, and metal abundance. From the results of the simulations, we synthesize the X-ray emission arising from the post-shock zone in the $[0.5-8.0] \mathrm{keV}$ band and in the resonance lines of the He-like ions $\mathrm{O}$ VII and Ne IX, taking into account the effect of the absorption from the surrounding stellar chromosphere.

The paper is organized as follow: Sect. 2 describes the numerical setup and the space of the physical parameters explored by the simulations; Sect. 3 reports the results focusing on the properties of the post-shock zone and on the X-ray emission; Sect. 4 discusses our results compared with the properties of CTTSs observed by high resolution X-ray spectrographs; in Sect. 5, we draw our conclusions.

\section{The Model}

\subsection{The numerical setup}

We adopt the model introduced in Paper I. We assume that accretion occurs along the magnetic flux tube linking the circumstellar disk to the star and that plasma moves and transports energy only along the magnetic field lines. This assumption is valid for values of the plasma parameter $\beta \ll 1$ (where $\beta=$ gas pressure/magnetic pressure) in the accretion column. We assume the accretion streams to be perpendicular to the stellar surface and focus our analysis on the portion of the stream close to the star with a constant mass accretion rate. This allows us to assume the plane-parallel geometry (the maximum expected ratio between the hot slab thickness and the stellar radius is $<0.2$ ). A schematic description of the system geometry is shown in Fig. 1. The model is one-dimensional and describes the impact of the stream onto the chromosphere along the coordinate s (see Fig. 1). The plasma dynamics is described by solving numerically the time-dependent fluid equations of mass, momentum, and energy conservation for a compressible conducting and optically thin plasma fluid above the stellar surface:

$$
\begin{aligned}
& \frac{\partial \rho}{\partial t}+\frac{\partial \rho u}{\partial s}=0 \\
& \frac{\partial \rho u}{\partial t}+\frac{\partial\left(P+\rho u^{2}\right)}{\partial s}=\rho g
\end{aligned}
$$

$\frac{\partial \rho E}{\partial t}+\frac{\partial(\rho E+P) u}{\partial s}=\rho u g+E_{\mathrm{H}}-\frac{\partial q}{\partial s}-n_{\mathrm{e}} n_{\mathrm{H}} \Lambda(T)$,

$$
\epsilon=\frac{P}{\rho(\gamma-1)}, \quad P=[1+\alpha(\rho, T)] \frac{\rho k_{\mathrm{B}} T}{\mu m_{\mathrm{H}}},
$$

where $t$ is the time; $s$ is the coordinate along the magnetic field lines (see Fig. 1); $u$ is the plasma velocity; $\rho=\mu m_{\mathrm{H}} n_{\mathrm{H}}$ is the mass density; $\mu$ is the mean atomic mass, that ranges between 1.277 and 1.381 times hydrogen mass as function of the adopted metal abundance; $m_{\mathrm{H}}$ is the mass of the hydrogen atom; $n_{\mathrm{e}}$ and $n_{\mathrm{H}}$ are the electron number density and the hydrogen number density, respectively; $P$ is the thermal pressure; $g(s)$ is the gravity of a star with a mass $M=0.8 M_{\odot}$ and a radius $R=1.3 R_{\odot} ; T$ is the plasma temperature; $E=\epsilon+u^{2} / 2$ is the total gas energy per unit mass; $\epsilon$ is the internal energy per unit mass; $\gamma=5 / 3$ is the ratio between specific heats; $k_{\mathrm{B}}$ is the Boltzmann constant; $q$ is the heat flux; $\Lambda(T)$ is the radiative losses function per unit emission measure; $\alpha(\rho, T)$ is the fractional ionization $\left(n_{\mathrm{e}} / n_{\mathrm{H}}\right)$, that has been derived from a modified Saha equation for the solar 


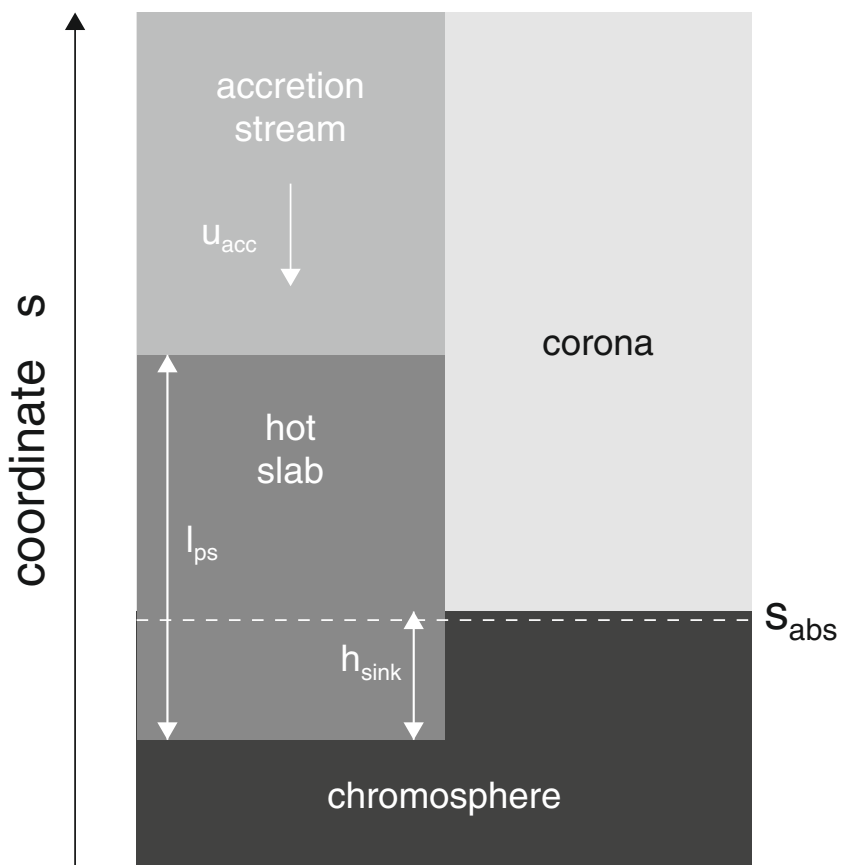

Fig. 1. Schematic description of the system geometry. $l_{\mathrm{ps}}$ and $h_{\text {sink }}$ are the expected thickness of the post-shock zone and the distance of the base of the slab from the transition region between the chromosphere and the stellar corona, respectively (see Sect. 2.2). $s_{\text {abs }}$ is the threshold below which, we assume the X-ray emission is fully absorbed (see Sect. 2.4).

chromosphere conditions (Brown 1973); $E_{\mathrm{H}}$ is a parametrized chromospheric heating function $\left(E_{\mathrm{H}}=0\right.$ for $\left.T>8 \times 10^{3} \mathrm{~K}\right)$ defined, as in Peres et al. (1982), to keep the unperturbed chromosphere in stable equilibrium.

The thermal conduction includes a smooth transition between the classical and the saturated conduction regime, as in Dalton \& Balbus (1993)

$q=\left(\frac{1}{q_{\mathrm{spi}}}+\frac{1}{q_{\mathrm{sat}}}\right)^{-1}$

where $q_{\text {spi }}=-\kappa(T) \partial T / \partial s$ is the classical conductive flux (Spitzer 1962) $\left(\right.$ with $\kappa(T)=9.2 \times 10^{-7} T^{5 / 2} \mathrm{erg} \mathrm{s}^{-1} \mathrm{~K}^{-1} \mathrm{~cm}^{-1}$ ), $q_{\mathrm{sat}}=-\operatorname{sign}(\partial T / \partial s) \phi \rho c_{\mathrm{s}}^{3}$ is the saturated flux (Cowie \& McKee 1977), $\phi \leq 1$ (Borkowski et al. 1989, and references therein) and $c_{\mathrm{S}}$ is the isothermal sound speed.

The radiative losses per unit of emission measure $\Lambda(T)$ have been calculated with the PINTofALE spectral code (Kashyap \& Drake 2000) and the APED V1.3 atomic line database (Smith et al. 2001). Since the radiative losses in the temperature range $10^{5}<T<10^{7} \mathrm{~K}$ are dominated by the emission lines from heavy ions, the radiative cooling depends on the metal abundance, $\zeta$, which is one of the parameters explored in this work.

The equations are solved numerically using the FLASH code (Fryxell et al. 2000) with its implementation of the PPM algorithm (Colella \& Woodward 1984). FLASH is a multi-physics code for solving astrophysical problems that handles an adaptive mesh by the PARAMESH algorithm (MacNeice et al. 2000). The code has been extended with an additional module for the evolution of the fractional hydrogen ionization $\left(n_{\mathrm{e}} / n_{\mathrm{H}}\right)$.

The extension of the computational domain depends on the specific parameters of the simulations (see Sect. 2.2). The largest domain considered here extends over a range $D=2.4 \times 10^{10} \mathrm{~cm}$ above the stellar surface, for the simulation that analyzes an

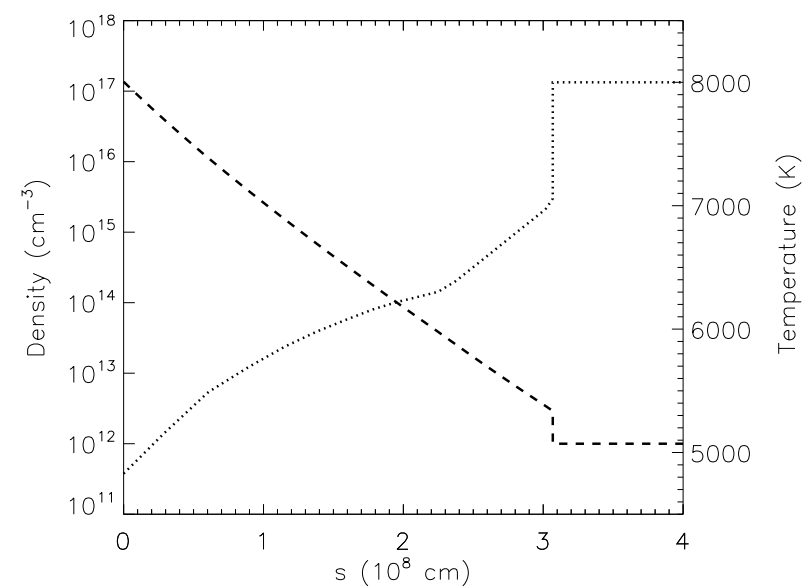

Fig. 2. Density (dashed line) and temperature (dotted line) profiles for the initial unperturbed stellar chromosphere $\left(s<3.05 \times 10^{8} \mathrm{~cm}\right)$ and an accretion stream $\left(s>3.05 \times 10^{8} \mathrm{~cm}\right)$ with hydrogen number density $n_{\text {acc }}=10^{12} \mathrm{~cm}^{-3}$ and solar metal abundance.

accretion stream density $n_{\mathrm{e}}=10^{10} \mathrm{~cm}^{-3}$, velocity $u_{\text {acc }}=$ $600 \mathrm{~km} \mathrm{~s}^{-1}$, and solar abundances (see Sect. 2.2 for a detailed description of the space of the physical parameters explored).

The spatial resolution adopted for each simulation depends on the thickness of the post-shock zone that spans a range of 6 orders of magnitude (See Sect. 3.1). At the highest resolution (stream with density $n_{\mathrm{e}}=10^{13} \mathrm{~cm}^{-3}$, velocity $u_{\mathrm{acc}}=200 \mathrm{~km} \mathrm{~s}^{-1}$ and metal abundance $\zeta=5$, in solar units) we allow for a maximum of 16 levels of refinement in the PARAMESH algorithm, with resolution increasing twice at each refinement level, and with the refinement criterion following the changes in density and temperature. This grid configuration yields an effective maximum resolution of $\approx 10^{3} \mathrm{~cm}$ at the finest level. For the simulation requiring the highest spatial resolution, we analyzed the effect of resolution on the model solution by considering additional simulations which use an identical setup but with a larger number of refinement levels. We checked that the resolution adopted in this paper is the best compromise between accuracy and computational cost and that the system evolution is well described in its details.

As initial condition we consider a stream with uniform density, temperature and velocity impacting onto a hydrostatic chromosphere (see Fig. 2). The temperature of the stream is the same in all the simulations performed and does not influence the system evolution, because the thermal energy of the stream is negligible with respect to its kinetic energy. The chromosphere of the young star is described adopting the solar models in Vernazza et al. (1973), rescaled to have, at its base, a pressure $\left(\approx 9 \times 10^{4} \mathrm{dyn}^{-2}\right)$ larger than the ram pressure of the streams considered here and an extension of $3 \times 10^{8} \mathrm{~cm}$. As boundary conditions, the values of density, temperature, and velocity are fixed to the initial ones both at the top (i.e. a constant accretion rate) and at the base of the computational domain. We checked that the adopted chromosphere and boundary conditions prevent boundary effects on the system evolution.

\subsection{Space of the physical parameters}

We explore the space defined by three parameters: the velocity and density of the pre-shock accretion flow, and the metal abundance of both the stellar and the accreting matter, that are assumed to be equal. Note however that a discrepancy between 
chromospheric and accretion metal abundances would not affect the results of our simulations, because, as discussed in Sacco et al. (2008) and in the Sect. 3.1 of this paper, the chromosphere acts as reservoir of matter stopping the accretion flow, but it does not influence the properties of the post-shock zone. Instead, different chromospheric abundances can slightly affect the absorption from the chromosphere as discussed in Sect. 2.4. We consider three pre-shock velocities $\left(u_{\mathrm{acc}}=200,400,600 \mathrm{~km} \mathrm{~s}^{-1}\right)$, 4 pre-shock densities $\left(n_{\text {acc }}=10^{10}, 10^{11}, 10^{12}, 10^{13} \mathrm{~cm}^{-3}\right)$ and 3 metal abundances $(\zeta=0.2,1.0,5.0$ in solar units retrieved from Anders \& Grevesse 1989). Both densities and metal abundances span larger ranges than those obtained from high spectral resolution X-ray observations. We focused our analysis on a large space of parameters for two main reasons. Probably the most important is that current high spectral resolution X-ray observations of CTTSs are limited to a very small sample of very close and bright CTTSs. These stars are older and accrete to a lower rate than typical CTTSs and it is most likely that physical and chemical properties of the corresponding accretion stream may differ too. Indeed, Bary et al. (2008) and Martin (1996) estimated pre-shock densities $n_{\mathrm{acc}}=10^{12}-10^{13} \mathrm{~cm}^{-3}$, while pre-shock densities derived from X-ray data are $n_{\text {acc }}=$ $10^{11}-10^{12} \mathrm{~cm}^{-3}$. Moreover, densities and metal abundances are measured from the spatially integrated emission including both the coronal and the accretion component. Typical coronal densities $\left(n_{\mathrm{e}}<10^{10} \mathrm{~cm}^{-3}\right)$ are much lower than densities expected in the accretion stream and metal abundances are lower than photospheric abundances observed in young stars (Telleschi et al. 2007). Therefore, both accretion stream densities and abundances derived from X-ray observations could be underestimated.

A heuristic model that assumes the strong shock approximation (Zel'Dovich \& Raizer 1967), stationary conditions and radiative cooling has been previously used to describe accretion shock physics in CTTSs (Lamzin 1998; Calvet \& Gullbring 1998) and to derive mass accretion rates from the X-ray emission (Argiroffi et al. 2007). This model provides post-shock region characteristics:

$$
\begin{aligned}
& u_{\mathrm{ps}}=\frac{u_{\mathrm{acc}}}{4}, \quad n_{\mathrm{ps}}=4 n_{\mathrm{acc}}, \\
& \tau_{\mathrm{cool}}=\frac{1}{\gamma-1} \frac{P}{n_{\mathrm{e}} n_{\mathrm{H}} \Lambda(T)} \sim 2.5 \times 10^{3} \frac{1}{\zeta} \frac{T_{\mathrm{ps}}^{3 / 2}}{n_{\mathrm{ps}}}, \\
& \tau_{\mathrm{cross}}=\frac{l_{\mathrm{ps}}}{u_{\mathrm{ps}}} \equiv \tau_{\mathrm{cool}}, \\
& T_{\mathrm{ps}}=\frac{3}{32} \frac{\mu m_{H}}{k_{\mathrm{B}}} u_{\mathrm{acc}}^{2} \approx 1.4 \times 10^{-9} u_{\mathrm{acc}}^{2}, \\
& l_{\mathrm{ps}} \equiv \tau_{\mathrm{cool}} u_{\mathrm{ps}}=1.7 \times 10^{-11} \frac{1}{\zeta} \frac{u_{\mathrm{acc}}^{4}}{n_{\mathrm{acc}}}
\end{aligned}
$$

where $u_{\mathrm{ps}}$ and $n_{\mathrm{ps}}$ are the post-shock velocity and density, $u_{\mathrm{acc}}$ and $n_{\text {acc }}$ are the stream pre-shock velocity and density, $\tau_{\text {cross }}$ is the crossing time of the accreting material through the postshock zone (which is expected to be equal to the radiative cooling time $\left.\tau_{\text {cool }}\right), T_{\mathrm{ps}}$ is the post-shock temperature, and $l_{\mathrm{ps}}$ is the thickness of the post-shock zone, and where the radiative cooling function has been approximated as $\Lambda(T) \approx 1.6 \times$ $10^{-19} \zeta T^{-1 / 2} \mathrm{erg} \mathrm{s}^{-1} \mathrm{~cm}^{3}$ (e.g. Orlando et al. 2005).

Table 1 reports the ranges of values of all the relevant physical parameters of the simulations explored here. The first three
Table 1. Range of relevant physical parameters explored.

\begin{tabular}{ccc}
\hline \hline Parameter & Range & Units \\
\hline$n_{\text {acc }}$ & $10^{10}-10^{13}$ & $\mathrm{~cm}^{-3}$ \\
$u_{\text {acc }}$ & $200-600$ & $\mathrm{~km} \mathrm{~s}^{-1}$ \\
$\zeta$ & $0.2-5.0$ & \\
\hline$T_{\mathrm{ps}}$ & $5.8 \times 10^{5}-5.6 \times 10^{6}$ & $\mathrm{~K}$ \\
$\tau_{\text {cool }}$ & $8.3 \times 10^{-3}-1000$ & $\mathrm{~s}$ \\
$l_{\mathrm{ps}}$ & $4.2 \times 10^{4}-1.5 \times 10^{10}$ & $\mathrm{~cm}$ \\
$P_{\text {ram }}$ & $35-7.8 \times 10^{4}$ & $\mathrm{dyn} \mathrm{cm}$ \\
$h_{\text {sink }}$ & $1.3 \times 10^{8}-3.5 \times 10^{8}$ & $\mathrm{~cm}$ \\
$\beta_{\mathrm{ps}}$ & $8.6 \times 10^{-4}-2.0$ & \\
\hline
\end{tabular}

Notes. $n_{\text {acc }}$ is the stream hydrogen number density; $u_{\text {acc }}$ is the stream velocity; $\zeta$ is the heavy element abundance; $T_{\mathrm{ps}}$ is the post-shock temperature; $\tau_{\text {cool }}$ is the radiative cooling time; $l_{\mathrm{ps}}$ is the expected thickness of the post-shock zone; $P_{\text {ram }}=\rho_{\text {acc }} u_{\text {acc }}^{2}$ is the ram pressure of the accretion flow; $h_{\text {sink }}$ is the sinking of the post-shock zone in the chromosphere; $\beta_{\mathrm{ps}}=P_{\mathrm{ram}} /\left(B^{2} / 8 \pi\right)$ is the plasma parameter in the post-shock zone, assuming a magnetic field $B=1 \mathrm{kG}$.

rows report the three independent parameters of our analysis (i.e. density, velocity, and metal abundance of the stream), whereas the following rows report the parameters derived from the independent ones, namely the post-shock temperature $T_{\mathrm{ps}}$ (Eq. (8)), the radiative cooling time $\tau_{\text {cross }}$ (Eq. (6)), the slab thickness $l_{\mathrm{ps}}$ (Eq. (9) and Fig. 1), the ram pressure of the accretion stream $P_{\text {ram }}=\rho_{\text {acc }} u_{\text {acc }}^{2}$, the sinking of the slab in the chromosphere $h_{\text {sink }}$ (i.e. the distance of the base of the slab from the transition region between the chromosphere and the stellar corona, see Fig. 1), and the plasma parameter of the post-shock zone $\beta=P_{\text {ram }} /\left(B^{2} / 8 \pi\right)$, where the magnetic field strength is assumed to be $B \sim 1 \mathrm{kG}$ (which is the order of magnitude of the field strengths derived from observations of CTTSs; JohnsKrull 2007). Note that the plasma $\beta$ is $\ll 1$ for most of the accretion streams considered in this work (thus justifying the low- $\beta$ assumption of our model) except for 5 cases, where $\beta \sim 1-2$ (for the magnetic field assumed). On the other hand, Orlando et al. (2010) showed that, for $\beta \sim 1-5$, the evolution of radiative shocks in 2D MHD models (thus including an explicit description of the ambient magnetic field) is analogous to that described by 1-D hydrodynamic models, although the amplitude of oscillations is smaller and the frequency higher than those predicted by 1-D models. For the 30 different cases analyzed here, the duration of the simulations ranges from 100 to $19000 \mathrm{~s}$. The duration of each simulation was set much longer than the initial transient effect, at the impact of the stream onto the chromosphere, and to include a large number (at least three) of oscillation periods of the post-shock zone (see Paper I).

\subsection{Synthesis of the $X$-ray emission}

For each simulation, we synthesize the X-ray luminosity $\left(L_{X}\right)$ in the $[0.5-8.0] \mathrm{keV}$ energy band and in the resonance lines of two He-like ions, namely the O VII at $21.60 \AA$ ( $\left.L_{\text {OVII }}\right)$ and the Ne IX at $13.45 \AA\left(L_{\mathrm{NeIX}}\right)$, which can be measured with the high resolution spectrographs on board the XMM-Newton and Chandra satellites. In particular:

1. From the spatial distributions of density and temperature (output of the numerical simulations), we compute the emission measure of the plasma in the $j$ th domain cell as $\mathrm{em}_{j}=$ $n_{\mathrm{H} j}^{2} A_{\mathrm{str}} \Delta s_{j}$, where $n_{\mathrm{H} j}$ is the particle number density in the 
cell, $\Delta s_{j}$ is the length of the $j$ th cell along the stream, and $A_{\text {str }}$ is the cross section of the stream. In this paper we assume $A_{\text {str }}=5 \times 10^{20} \mathrm{~cm}^{2}$, (i.e. $\sim 0.5 \%$ of the stellar surface); for the values of stream velocity and density explored here, this cross section produces mass accretion rates spanning the range of values derived from X-ray observations of CTTSs (see Sect. 3.3). We then derive the distribution of emission measure versus temperature, $\operatorname{EM}(T)$, by binning the emission measure values into slots of temperature; the range of temperature $[4<\log T(\mathrm{~K})<8]$ is divided into 81 bins, all equal on logarithmic scale $(\Delta \log (T)=0.05)$.

2. From the $\operatorname{EM}(T)$ distributions, we synthesize the $\mathrm{X}$-ray emission in the [0.5-8.0] $\mathrm{keV}$ band and in the resonance lines of $\mathrm{O}$ VII and Ne IX, considering the appropriate metal abundance $\zeta$, using the PINTofALE spectral code with the APED V1.3 atomic line database.

3. Finally, we derive time-average luminosities $L_{\mathrm{X}}, L_{\mathrm{OVII}}, L_{\mathrm{NeIX}}$ over a selected time interval including, at least, three oscillation cycles and outside initial transients.

\subsection{Absorption from the stellar atmosphere}

As pointed out by Drake (2005), a large fraction of the X-ray emission due to the accretion shock may be absorbed by the gas present in the surrounding stellar atmosphere or by the accretion column itself. The absorption from the stellar atmosphere mainly depends on the location of the emitting plasma. For a given shocked slab of material rooted in the chromosphere, photons emitted from plasma located at the base of the slab (deep into the chromosphere) travel through higher density gas layers than photons from plasma located in the shallower portion of the slab. The deepness of the emitting plasma in the chromosphere depends on the thickness of the slab $l_{\mathrm{ps}}$ and on the sinking of the slab in the chromosphere $h_{\text {sink }}$ (Sect. 2.2) to the position at which the ram pressure of the post-shock plasma equals the thermal pressure of the chromosphere. As discussed in Sect. 2.2, our simulations explore a wide range of values for $l_{\mathrm{ps}}$ and $h_{\text {sink }}$ (see Table 1).

In order to estimate the effect of the absorption on the observed X-ray emission, we calculate the X-ray luminosities by assuming that only the emission from plasma located in the shallower layers of the shocked slab can be observed (see also Drake 2005 for a previous application of this method to estimate absorption effects). Specifically, we assume that all the emission due to plasma located at $s<s_{\text {abs }}$ is fully absorbed, while the emission produced from plasma located at $s>s_{\text {abs }}$ is fully transmitted. The threshold $s_{\mathrm{abs}}$ is defined as the height, in the unperturbed stellar chromosphere, at which the overlying atmosphere absorbs $50 \%$ of the energy of a trial X-ray spectrum (see also Fig. 1). We considered as trial X-ray spectrum that produced by a plasma at $T=1 \mathrm{MK}$. The plasma temperature does not influence the threshold $s_{\text {abs }}$ used for calculating the Ne IX and the O VIII resonance line luminosities, while it slightly influences the thresholds used for the $[0.5-8.0] \mathrm{keV}$ band. However, we found that the results of only a few of the parameter configurations considered here, specifically those leading to $l_{\mathrm{ps}} \sim h_{\text {sink }}$, slightly depend on the particular choice of the trial X-ray spectrum. For these configurations a more detailed description of the absorption effect, including the dependence on the wavelength, a more smooth transition between the full absorption and the full transmission and a dependence of the angle of view should be performed to give a more precise answer to the observability issue.
Table 2. Values of the threshold $s_{\text {abs }}$ as a function of the energy band and of the metal abundance.

\begin{tabular}{cccc}
\hline \hline$\zeta$ & $\begin{array}{c}{[0.5-8.0] \mathrm{keV}} \\
\left(10^{8} \mathrm{~cm}\right)\end{array}$ & $\begin{array}{c}\text { O VII } \\
\left(10^{8} \mathrm{~cm}\right)\end{array}$ & $\begin{array}{c}\text { Ne IX } \\
\left(10^{8} \mathrm{~cm}\right)\end{array}$ \\
\hline 0.2 & 3.56 & 3.57 & 3.17 \\
1.0 & 3.72 & 3.74 & 3.43 \\
5.0 & 3.81 & 3.83 & 3.58 \\
\hline
\end{tabular}

The values of $s_{\mathrm{abs}}$ for the three X-ray bands and for the three metal abundances are reported in Table 2. The value of $s_{\text {abs }}$ obviously depends on the chromospheric metal abundances, that we assume to be equal to the abundances of the accreting material. However, chromospheric abundances may be different from accretion stream abundances. For instance, if the chromospheric abundance is higher or lower than the accretion stream abundance, then we underestimate or overestimate the absorption effect, respectively. A better knowledge of metal abundances of the different components of the star-disk system is required to better address this issue.

We found that $s_{\text {abs }}$ does not change significantly if we consider the absorbed X-ray emission in the [0.5-8.0] keV band or in the O VII resonance line, because the absorption in the softer part of the [0.5-8.0] keV band (which is the same of the O VII line) is dominant. On the other hand, we found a significantly lower value of $s_{\mathrm{abs}}$ in the Ne IX resonance line, this line forming at higher energy (where the absorption is lower) than O VII. Therefore, we consider one set of thresholds $s_{\text {abs }}$ for the synthesis of the luminosities in the $[0.5-8.0] \mathrm{keV}$ band and in the O VII resonance line, and another set of $s_{\text {abs }}$ for the luminosities in the Ne IX resonance line (see Table 2).

\section{Results}

\subsection{Physical properties of the post-shock zone}

In all the simulations, during the first 100-200 s, the system follows the same evolution as described in Paper I. Figure 3 shows, as an example, the evolution of plasma temperature, density, pressure and velocity for a stream with $n_{\mathrm{acc}}=10^{11} \mathrm{~cm}^{-3}$, $u_{\mathrm{acc}}=400 \mathrm{~km} \mathrm{~s}^{-1}$ and $\zeta=1.0$. The accretion stream penetrates the chromosphere and generates a transmitted shock and a reverse shock. After this transient phase, the chromosphere stops the flow penetration where the ram pressure of the post-shock plasma equals the thermal pressure of the chromosphere. The reverse shock progressively builds up a nearly isothermal slab at the post-shock temperature (heating phase; left panels of Fig. 3). During this phase the intensity of the radiative cooling at the base of the slab gradually increases. The heating phase ends when the radiative cooling triggers a thermal instability that robs the postshock plasma of pressure support, causing the material above the cooled layer to collapse back (cooling phase; right panels of Fig. 3). Consequently the reverse shock moves downwards to the chromosphere reducing the post-shock zone thickness. After the hot slab has disappeared, a new slab is re-built by the reverse shock, starting a new cycle of quasi-periodic shock oscillations. The post-shock zone is separated from the cold chromosphere by a very steep transition region located where the chromospheric thermal pressure equals the ram pressure of the post-shock plasma. The thermal conduction drains energy from the shock-heated plasma to the chromosphere through this transition region, acting as an additional cooling mechanism (see also Orlando et al. 2010). The effects of the heat conduction are 


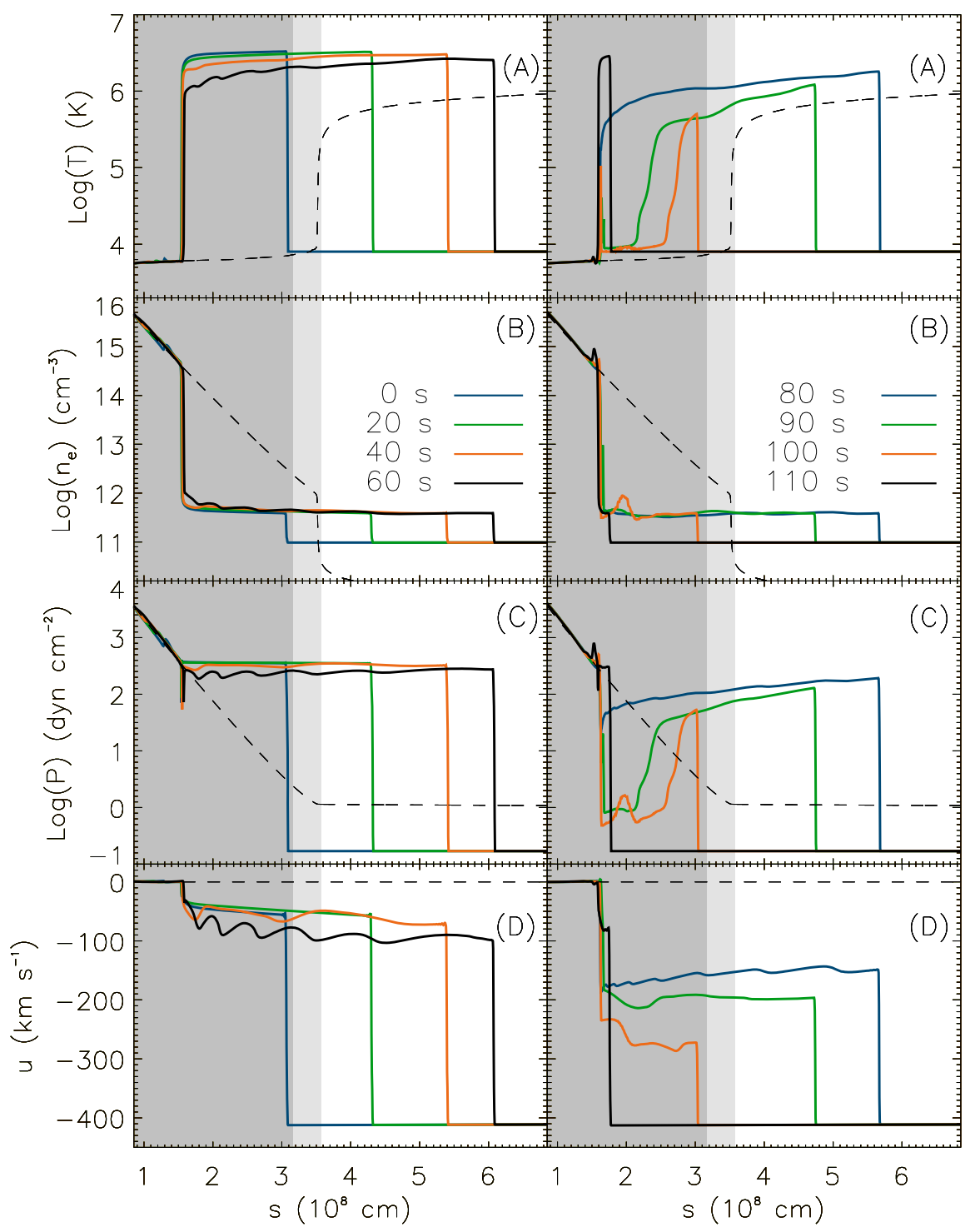

Fig. 3. Time evolution of plasma temperature A), density B), pressure C) and velocity D) during a complete oscillation of the post-shock zone in the case of an accretion stream with density $n_{\text {acc }}=10^{11} \mathrm{~cm}^{-3}$, velocity $u_{\mathrm{acc}}=$ $400 \mathrm{~km} \mathrm{~s}^{-1}$ and metal abundance $\zeta=1.0$. The left (right) panels show the time evolution during the heating (cooling) phase. The dashed lines describe the profiles of the unperturbed initial stellar atmosphere (chromosphere and corona). The shaded areas mark the regions below the thresholds $s_{\text {abs }}$ used to estimate the effects of absorption on the luminosity in the $\mathrm{O}$ VII (pale grey) and Ne IX resonance lines (see Sect. 2.4). the largest in the simulations with the highest post-shock temperatures (i.e. the highest stream velocities).

Figure 4 and Table 3 show the main physical parameters characterizing the structure of the post-shock zone after the initial transient phase, namely the maximum extension of the postshock slab $\left(l_{\max }\right)$, the oscillation period $\left(P_{\mathrm{osc}}\right)$, and the emissionmeasure-weighted temperature $\left(T_{\mathrm{ps}}\right)$ of the post-shock zone. In order to define $l_{\max }$, we consider all the material hotter than $T=3 \times 10^{5} \mathrm{~K}$ as shocked plasma. The steepness of the transition region makes $l_{\max }$ poorly sensitive to this choice. The periods of the oscillations have been determined through a Fourier analysis of the length of the post-shock zone as a function of time. The temperature $T_{\mathrm{ps}}$ has been calculated from the emission measure distribution $\operatorname{EM}(T)$ (see Sect. 2.3), considering only bins with $\log T(\mathrm{~K})>5$.

The maximum extension of the post-shock zone ranges between a factor 0.3 and 1.7 of the length estimated using Eq. (9), whereas the post-shock temperature ranges between a factor 0.7 and 1.0 of the temperature estimated using Eq. (8). The simulated post-shock temperatures are slightly lower than those derived from Eq. (8) because the former are averaged over the whole post-shock zone, whereas the latter are estimated at the shock front, at the maximum extension of the slab. In addition, the simulated post-shock temperatures are averaged over several shock oscillations, including both the heating and the cooling phase of the evolution. During the former, the temperature is slightly higher than that derived from Eq. (8), because the plasma velocity in the reference frame of the shock is higher than $u_{\text {acc }}$; the opposite is true during the cooling phase.

The post-shock temperature depends on the accretion stream velocity, whereas both the oscillation period $P_{\mathrm{osc}}$ and the length of the post-shock zone $l_{\max }$ depend on the whole set of model parameters (velocity, density and metal abundance of the stream). Figure 4 and Table 3 show that $P_{\text {osc }}$ and $l_{\max }$ both span 5-6 orders of magnitude for the range of parameters explored here. In the complex magnetospheric accretion scenario, the mass density and velocity of the flow could vary along the accretion stream cross section (see for instance Romanova et al. 2004). Consequently, in the case of $\beta \ll 1$, an accretion stream cannot be described by a single $1-\mathrm{D}$ model but has to be considered as a bundle of independent fibrils, each described in terms of a different 1-D model, and each independent on the others (with different instability periods and random phases of the shock oscillations) due to the strong magnetic field which prevents mass and energy exchange across magnetic field lines. We expect therefore that accretion streams consisting of many 
Table 3. Physical properties of the post-shock zone and X-ray luminosities.

\begin{tabular}{|c|c|c|c|c|c|c|c|c|c|c|c|}
\hline $\begin{array}{c}n_{\mathrm{acc}} \\
\left(\mathrm{cm}^{-3}\right)\end{array}$ & $\begin{array}{c}u_{\mathrm{acc}} \\
\left(\mathrm{km} \mathrm{s}^{-1}\right)\end{array}$ & $\zeta$ & $\begin{array}{c}P_{\text {osc }} \\
(\mathrm{s})\end{array}$ & $\begin{array}{l}l_{\max } \\
(\mathrm{cm})\end{array}$ & $\begin{array}{c}T_{\mathrm{ps}} \\
(\mathrm{MK})\end{array}$ & $\begin{array}{c}\log \left(L_{\mathrm{X}}\right)^{a} \\
\left(\mathrm{erg} \mathrm{s}^{-1}\right)\end{array}$ & $\begin{array}{c}\log \left(L_{X}\right)^{b} \\
\left(\operatorname{erg~s}^{-1}\right)\end{array}$ & $\begin{array}{c}\log \left(L_{\mathrm{OVII}}\right)^{c} \\
\left(\mathrm{erg} \mathrm{s}^{-1}\right)\end{array}$ & $\begin{array}{c}\log \left(L_{\mathrm{OVIII}}\right)^{d} \\
\left(\mathrm{erg} \mathrm{s}^{-1}\right)\end{array}$ & $\begin{array}{c}\log \left(L_{\text {NeIX }}\right)^{e} \\
\left(\mathrm{erg} \mathrm{s}^{-1}\right)\end{array}$ & $\begin{array}{c}\log \left(L_{\mathrm{NeIX}}\right)^{f} \\
\left(\mathrm{erg} \mathrm{s}^{-1}\right)\end{array}$ \\
\hline $10^{10}$ & 400 & 1.0 & $1.3 \times 10^{3}$ & $5.0 \times 10^{9}$ & 2.3 & 28.41 & 28.40 & 27.60 & 27.60 & 26.79 & 26.79 \\
\hline $10^{10}$ & 400 & 5.0 & $5.0 \times 10^{2}$ & $6.0 \times 10^{8}$ & 2.0 & 28.20 & 28.11 & 27.58 & 27.46 & 26.45 & 26.43 \\
\hline $10^{10}$ & 600 & $\begin{array}{l}5.0 \\
1.0\end{array}$ & $3.8 \times 10^{3}$ & $1.5 \times 10^{10}$ & 4.1 & 29.32 & 29.32 & 28.04 & 28.00 & 27.66 & 27.66 \\
\hline $10^{10}$ & 600 & 5.0 & $1.2 \times 10^{3}$ & $5.0 \times 10^{9}$ & 4.6 & 29.51 & 29.48 & 28.11 & 28.00 & 27.81 & 27.79 \\
\hline $10^{11}$ & 200 & 0.2 & $6.1 \times 10^{1}$ & $8.0 \times 10^{7}$ & 0.5 & 25.52 & 25.30 & 24.90 & 24.68 & 21.67 & 21.67 \\
\hline $10^{11}$ & 200 & 1.0 & $1.5 \times 10^{1}$ & $2.0 \times 10^{7}$ & 0.5 & 25.75 & - & 25.18 & - & 22.08 & 21.36 \\
\hline $10^{11}$ & 200 & 5.0 & $3.2 \times 10^{0}$ & $4.2 \times 10^{6}$ & 0.5 & 25.67 & - & 25.11 & - & 21.97 & $\begin{array}{l}21.50 \\
-\end{array}$ \\
\hline $10^{11}$ & 400 & 0.2 & $5.0 \times 10^{2}$ & $1.5 \times 10^{9}$ & 2.1 & 29.30 & 29.26 & 28.53 & 28.49 & 27.56 & 27.54 \\
\hline $10^{11}$ & 400 & 1.0 & $1.6 \times 10^{2}$ & $4.0 \times 10^{8}$ & 2.1 & 29.18 & 28.89 & 28.49 & 28.18 & 27.46 & 27.32 \\
\hline $10^{11}$ & 400 & 5.0 & $4.2 \times 10^{1}$ & $5.5 \times 10^{7}$ & 2.1 & 29.00 & $\begin{array}{c}20.09 \\
-\end{array}$ & 28.36 & $\begin{array}{c}20.10 \\
-\end{array}$ & 27.23 & $\begin{array}{l}21.02 \\
-\end{array}$ \\
\hline $10^{11}$ & 600 & 0.2 & $1.3 \times 10^{3}$ & $7.0 \times 10^{9}$ & 4.4 & 30.36 & 30.34 & 28.89 & 28.85 & 28.54 & 28.53 \\
\hline $10^{11}$ & 600 & $\begin{array}{l}0.2 \\
1.0\end{array}$ & $5.0 \times 10^{2}$ & $2.5 \times 10^{9}$ & $\begin{array}{l}4.4 \\
4.6\end{array}$ & 30.40 & 30.34 & $\begin{array}{l}20.09 \\
28.96\end{array}$ & $\begin{array}{l}20.0 J \\
28.88\end{array}$ & $\begin{array}{l}20.04 \\
28.65\end{array}$ & 28.61 \\
\hline $10^{11}$ & 600 & 5.0 & $3.8 \times 10^{2}$ & $3.7 \times 10^{8}$ & 4.9 & 30.18 & 29.72 & 28.59 & $\begin{array}{l}20.00 \\
27.72\end{array}$ & 28.51 & 28.04 \\
\hline $10^{12}$ & 200 & 0.2 & $5.7 \times 10^{0}$ & $6.2 \times 10^{6}$ & 0.5 & 26.30 & $\begin{array}{c}29.12 \\
-\end{array}$ & 25.69 & $\begin{array}{l}21.12 \\
-\end{array}$ & 22.38 & $\begin{array}{c}20.04 \\
-\end{array}$ \\
\hline $10^{12}$ & 200 & $\begin{array}{l}0.2 \\
1.0\end{array}$ & $1.4 \times 10^{0}$ & $1.7 \times 10^{6}$ & 0.5 & 26.54 & - & 25.96 & - & $\begin{array}{l}22.50 \\
22.76\end{array}$ & - \\
\hline $10^{12}$ & 200 & $\begin{array}{l}1.0 \\
5.0\end{array}$ & $2.8 \times 10^{-1}$ & $3.7 \times 10^{5}$ & 0.5 & $\begin{array}{l}20.04 \\
26.51\end{array}$ & - & 25.92 & - & 22.66 & - \\
\hline $10^{12}$ & 400 & 0.2 & $4.7 \times 10^{1}$ & $5.0 \times 10^{7}$ & 1.9 & 30.08 & - & 29.46 & - & 28.18 & 26.28 \\
\hline $10^{12}$ & 400 & 1.0 & $1.2 \times 10^{1}$ & $1.6 \times 10^{7}$ & 1.9 & 30.18 & - & 29.54 & - & 28.43 & - \\
\hline $10^{12}$ & 400 & 5.0 & $2.9 \times 10^{0}$ & $5.0 \times 10^{6}$ & 2.0 & 29.68 & - & 29.08 & - & 27.88 & - \\
\hline $10^{12}$ & 600 & 0.2 & $3.3 \times 10^{2}$ & $5.5 \times 10^{8}$ & 4.4 & 31.11 & 30.72 & 29.62 & 28.91 & 29.36 & 29.04 \\
\hline $10^{12}$ & 600 & 1.0 & $1.5 \times 10^{2}$ & $1.5 \times 10^{8}$ & 4.6 & 31.11 & - & 29.56 & - & 29.48 & - \\
\hline $10^{12}$ & 600 & 5.0 & $2.9 \times 10^{1}$ & $2.5 \times 10^{7}$ & 4.8 & 31.08 & - & 29.49 & - & 29.43 & - \\
\hline $10^{13}$ & 200 & 0.2 & $5.9 \times 10^{-1}$ & $3.5 \times 10^{5}$ & 0.5 & 27.43 & - & 26.81 & - & 23.60 & - \\
\hline $10^{13}$ & 200 & 1.0 & $1.4 \times 10^{-1}$ & $1.5 \times 10^{5}$ & 0.5 & 27.15 & - & 26.53 & - & 23.11 & - \\
\hline $10^{13}$ & 200 & 5.0 & $2.8 \times 10^{-2}$ & $3.2 \times 10^{4}$ & 0.5 & 27.49 & - & 26.91 & - & 23.63 & - \\
\hline $10^{13}$ & 400 & 0.2 & $4.1 \times 10^{0}$ & $5.0 \times 10^{6}$ & 1.8 & 31.11 & - & 30.43 & - & 29.26 & - \\
\hline $10^{13}$ & 400 & 1.0 & $2.2 \times 10^{0}$ & $1.5 \times 10^{6}$ & 2.1 & 30.91 & - & 30.32 & - & 29.04 & - \\
\hline $10^{13}$ & 400 & 5.0 & $3.2 \times 10^{0}$ & $3.4 \times 10^{5}$ & 1.9 & 30.63 & - & 30.04 & - & 28.80 & - \\
\hline $10^{13}$ & 600 & 0.2 & $8.3 \times 10^{1}$ & $3.8 \times 10^{7}$ & 4.4 & 32.08 & - & 30.45 & - & 30.36 & - \\
\hline $10^{13}$ & 600 & 1.0 & $7.3 \times 10^{1}$ & $1.3 \times 10^{7}$ & 4.4 & 32.23 & - & 30.76 & - & 30.64 & - \\
\hline
\end{tabular}

Notes. ${ }^{(a)}$ Luminositity in the $0.5-8.0 \mathrm{keV}$ band not considering the absorption. ${ }^{(b)}$ Luminositity in the $0.5-8.0 \mathrm{keV}$ band considering the absorption from the stellar atmosphere as discussed in the text. ${ }^{(c)}$ Luminositity in the O VII resonance line at $21.6 \AA$ not considering the absorption. ${ }^{(d)}$ Luminositity in the O VII resonance line at $21.6 \AA$ considering the absorption from the stellar atmosphere as discussed in the text. ${ }^{(e)}$ Luminositity in the Ne IX resonance line at $13.45 \AA$ not considering the absorption. ${ }^{(f)}$ Luminositity in the Ne IX resonance line at $13.45 \AA$ considering the absorption from the stellar atmosphere as discussed in the text.

(200-300) different fibrils with different instability periods and random phases would produce no periodic variations of the $\mathrm{X}$ ray emission from shocked plasma, as recently found in the case of TW Hydrae (Drake et al. 2009).

As discussed in Sect. 2.4, the key parameters for understanding the effects of the absorption by the stellar chromosphere on the X-ray emission from shocked accreted plasma are the thickness of the shocked slab $l_{\max }$ and the sinking of the slab in the chromosphere to the position $h_{\text {sink }}$ at which the ram pressure of the post-shock plasma equals the thermal pressure of the chromosphere. Figure 4 shows that $l_{\max }$ is anti-correlated with the density of the accretion stream and with the metal abundance, and is correlated with the stream velocity (see also Eq. (9)). On the other hand, $h_{\text {sink }}$ is correlated with both the stream density and velocity, the ram pressure of the post-shock plasma being $P_{\text {ram }} \propto \rho_{\text {acc }} u_{\text {acc }}^{2}$ and the thermal pressure of the chromosphere decreasing with height. Consequently, the shocked slab from high density streams with high metal abundances is expected to hardly emerge from the dense chromospheric layers, where the absorption is strong, because the post-shock zone is rooted deeply in the chromosphere ( $h_{\text {sink }}$ is large) and the slab is rather thin $\left(l_{\max }\right.$ is small). On the other hand, streams with high velocity form extended post-shock zones (being $l_{\max } \propto u_{\text {acc }}^{4}$ ) and the shocked slab may easily emerge above the chromosphere even if it is deeply rooted in the chromosphere, the ram pressure being proportional to the square of velocity.

\subsection{Distribution of emission measure versus temperature}

The distribution of emission measure versus temperature $\operatorname{EM}(T)$ of the shock-heated plasma is a useful source of information of the plasma components contributing to the X-ray emission and is directly comparable with $\operatorname{EM}(T)$ distributions derived from X-ray observations (see, for instance, Argiroffi et al. 2009). Figure 5 shows how the time-averaged $\operatorname{EM}(T)$ of the post-shock plasma varies as a function of velocity (upper panel), density (middle panel), and metal abundance (lower panel) of the accretion stream. The case with density $n_{\mathrm{acc}}=10^{12} \mathrm{~cm}^{-3}$, velocity $u_{\mathrm{acc}}=400 \mathrm{~km} \mathrm{~s}^{-1}$ and solar abundance $\zeta=1$ (red dotted line in Fig. 5) is the reference in all the panels. In all cases, our model predicts a monolithic distribution of emission measure of the post-shock plasma that covers the entire range of values below the temperature of the shock front. The ascending part of the $\operatorname{EM}(T)$ distribution corresponds to cooled postshock plasma of the slab and to the transition region between the shocked chromosphere and the hot slab. This characteristic of the $\operatorname{EM}(T)$ distribution cannot be reproduced by heuristic models 


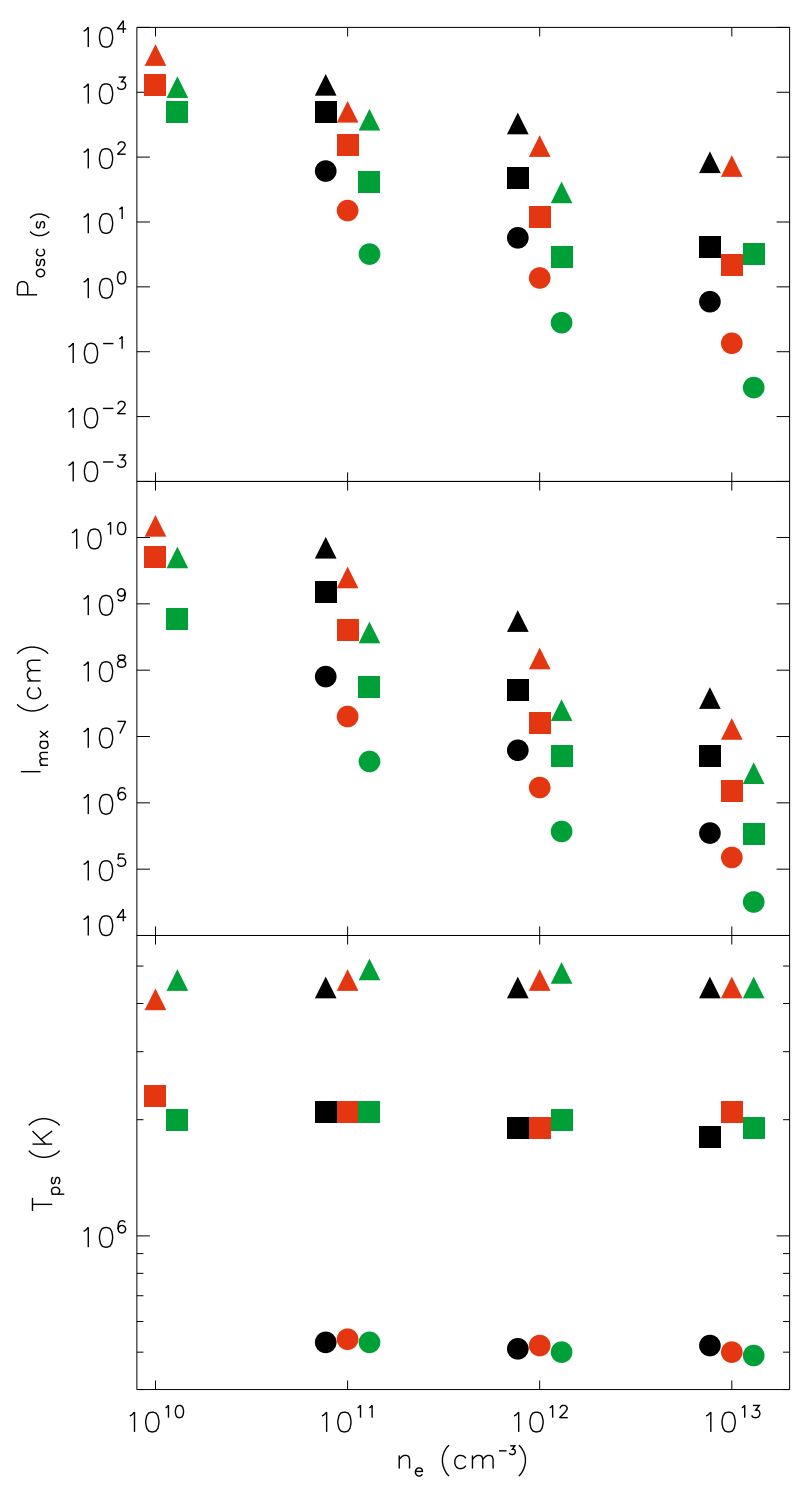

Fig. 4. Periods of the shock oscillations ( $P_{\text {osc }}$, upper panel $)$, maximum extension of the post-shock zone ( $l_{\max }$, middle panel) and the emissionmeasure-weighted temperature $\left(T_{\mathrm{ps}}\right.$, bottom panel $)$ of all the cases investigated. The labels on the $x$-axis of the bottom panel indicate the densities of accretion stream. Different symbols indicate different velocities: $u_{\mathrm{acc}}=200$ (circle), 400 (squares), and 600 (triangles) $\mathrm{km} \mathrm{s}^{-1}$. Different colors indicate different metal abundances: $\zeta=0.2$ (black), 1.0 (red) and 5.0 (green). Note that data points have been spread over each density value to prevent overlapping of the symbols.

(e.g. Lamzin 1998; Calvet \& Gullbring 1998; Argiroffi et al. 2007) and has been proved to be in agreement with observations (e.g. Argiroffi et al. 2009).

The total emission measure of plasma above $1 \mathrm{MK}$ and the maximum temperature of the emission measure distribution depend on the velocity of the accretion stream (upper panel in Fig. 5). In fact, the temperature of the post-shock plasma depends on the square of the stream velocity (see Eq. (8)), leading to a shift of the $\operatorname{EM}(T)$ profile towards higher temperature for higher value of $u_{\text {acc }}$. The thickness of the post-shock zone strongly increases with the stream velocity (see Fig. 4 and Eq. (9)), so that its volume and therefore the overall emission measure of the shock-heated plasma increases with $u_{\text {acc }}$.

Not surprisingly, the density of the stream determines the overall emission measure in the hot slab (see middle panel in

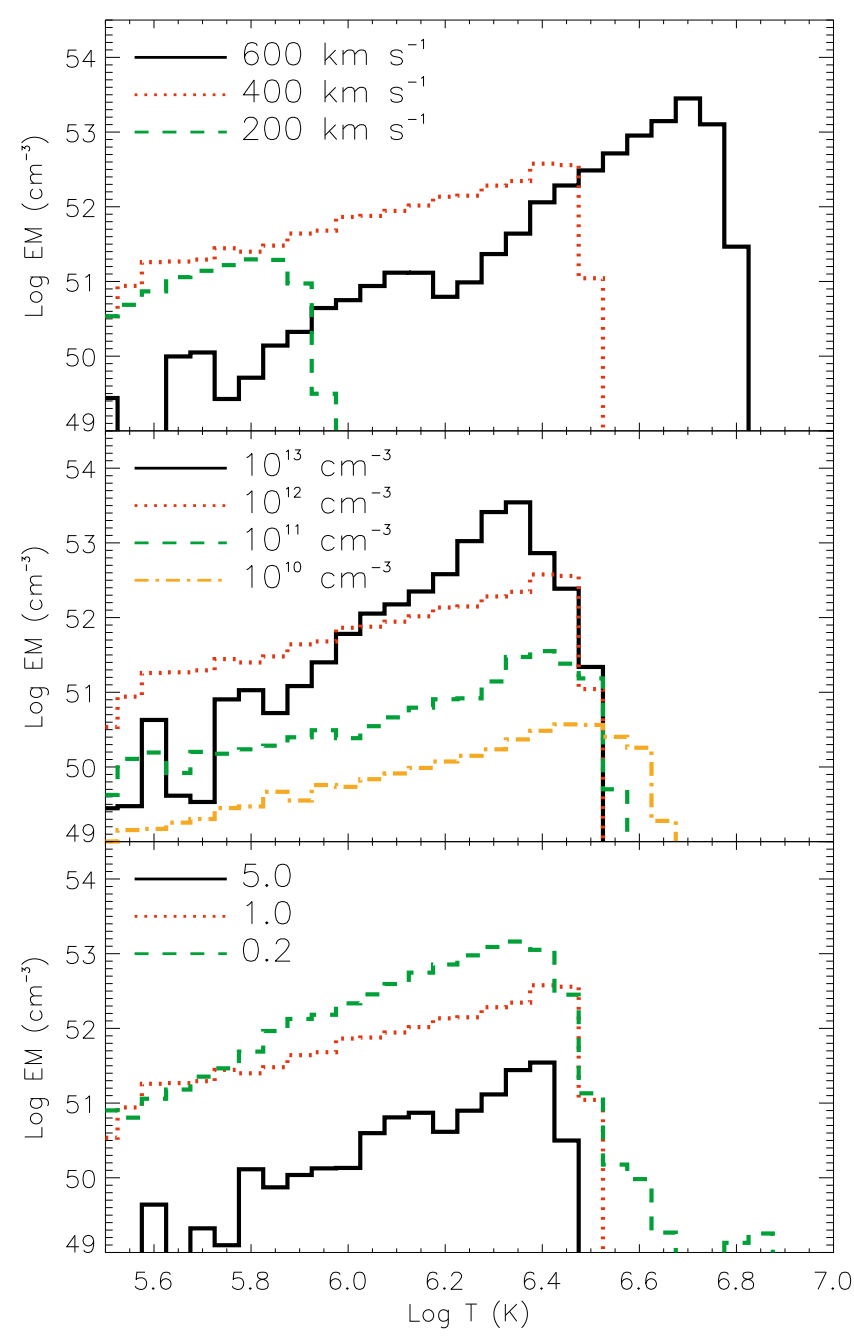

Fig. 5. Time-averaged emission measure distribution of the post-shock plasma for accretion streams with the same metal abundance $(\zeta=1.0)$ and density $\left(n_{\text {acc }}=10^{12} \mathrm{~cm}^{-3}\right)$ but different velocities (upper panel), for streams with the same metal abundance $(\zeta=1.0)$ and velocity $\left(u_{\text {acc }}=\right.$ $400 \mathrm{~km} \mathrm{~s}^{-1}$ ) but different densities (middle panel), and for streams with the same density $\left(n_{\text {acc }}=10^{12} \mathrm{~cm}^{-3}\right)$ and velocity $\left(u_{\mathrm{acc}}=400 \mathrm{~km} \mathrm{~s}^{-1}\right)$ but different metal abundances (lower panel).

Fig. 5): the higher the stream density, the higher the emission measure of the slab. Note however that, since $l_{\text {acc }} \propto n_{\text {acc }}^{-1}$ (see Eq. (9)), the emission measure of the post-shock zone depends only linearly on $n_{\text {acc }}$ (not on its square). Figure 5 also shows that the ascending branch of the $\operatorname{EM}(T)$ distribution is steeper for larger values of density.

Finally, the $\operatorname{EM}(T)$ distribution depends on the metal abundance: the higher the value of $\zeta$, the lower the emission measure of the slab. In fact, the length of the post-shock zone depends inversely on the metal abundance (see Eq. (9)) as a consequence of more efficient radiative cooling for larger $\zeta$. Consequently, the emission measure of the hot slab decreases for increasing $\zeta$.

\subsection{X-ray emission}

$\mathrm{X}$-ray luminosities derived for each simulation are reported in Table 3 and in the Fig. 6, where they are plotted against the mass accretion rate derived assuming the same accretion stream cross section $\left(A_{\text {str }}=5 \times 10^{20} \mathrm{~cm}^{2}\right)$ used for deriving emission measure distribution (see Sect. 2.3). The left panels show the 


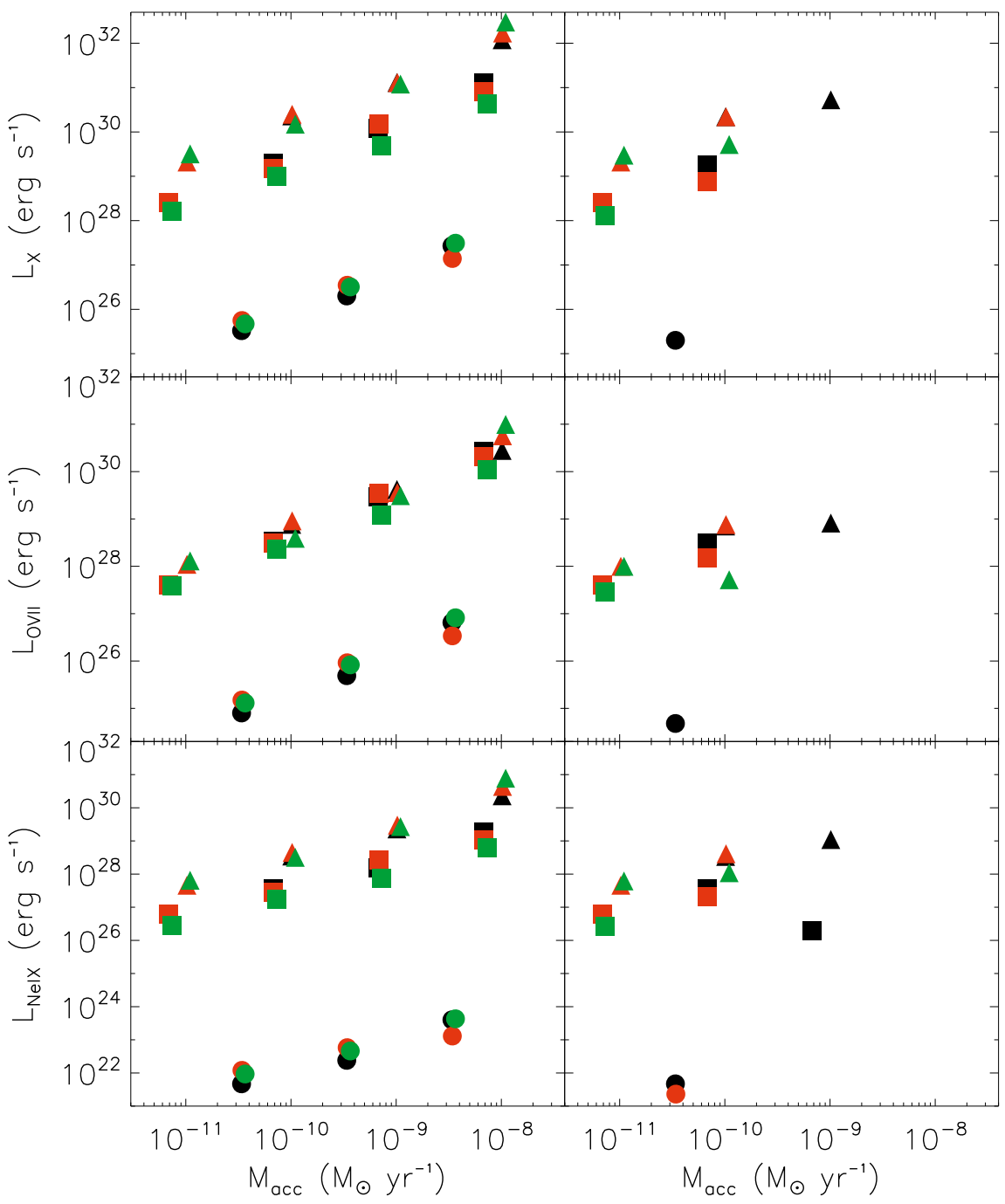

Fig. 6. Luminosities in the $[0.5-8.0] \mathrm{keV}$ band (top panels) and in the resonance lines of the $\mathrm{O}$ VII (middle-panels) and Ne IX (bottom pan$e l s$ ) as function of the mass accretion rates. Absorption from the stellar chromosphere is considered only for the results reported on right panels. Symbols and colors as in Fig. 4. results obtained not considering absorption due to the stellar atmosphere, whereas right panels show the results including the absorption effects using the method discussed in Sect. 2.4.

The dependence of the X-ray luminosities on the model parameters can be easily explained by interpreting the results shown in the right panels of Fig. 6 in the light of the $\operatorname{EM}(T)$ distributions shown in Fig. 5 and of the relations between the main properties of the post-shock zone and the stream parameters in Fig. 4. The spread in the X-ray luminosity due to the metal abundance is less than 0.5 dex even with the large range (a factor 25) of metal abundances considered in this work. This result is due to two different effects working in opposite directions. $\operatorname{The} \operatorname{EM}(T)$ of the post-shock plasma is anti-correlated with the metal abundance (bottom panel of Fig. 5), but higher metal abundances trigger higher emission in the soft X-ray band, because soft X-ray emission is mainly due to line emission produced by heavy ions.

The X-ray luminosity is strongly correlated with the accretion stream velocity: $X$-ray luminosities derived for streams at velocities $u_{\mathrm{acc}}=400 \mathrm{~km} \mathrm{~s}^{-1}$ and $u_{\mathrm{acc}}=600 \mathrm{~km} \mathrm{~s}^{-1}$ differ for about one order of magnitude, whereas X-ray luminosities produced by streams with $u_{\text {acc }}=200 \mathrm{~km} \mathrm{~s}^{-1}$ are more than three orders of magnitude lower than the others. In addition, the spread between the streams at 400 and $600 \mathrm{~km} \mathrm{~s}^{-1}$ is much less in the O VII resonance line (left-central panel of Fig. 6) than in the
[0.5-8.0] keV band. Evidently, the relation between X-ray luminosity and stream velocity strongly depends on the profile of the $\operatorname{EM}(T)$ distribution. In fact, low velocity $\left(200 \mathrm{~km} \mathrm{~s}^{-1}\right) \mathrm{ac}-$ cretion streams cannot heat up plasma to temperatures greater than $1 \mathrm{MK}$ (top panel of Fig. 5), so that the accretion energy is emitted mainly in the extreme ultraviolet band and very faint Xray emission is expected. The peak of the $\operatorname{EM}(T)$ for the stream at $400 \mathrm{~km} \mathrm{~s}^{-1}(\log T(\mathrm{~K})=6.4)$ is very close to the peak of the emissivity function of the $\mathrm{O}$ VII resonance line and it is comparable to the EM value at $\log T(\mathrm{~K})=6.4$ for the case at $600 \mathrm{~km} \mathrm{~s}^{-1}$, explaining the small difference between the O VII luminosities for streams at 400 and $600 \mathrm{~km} \mathrm{~s}^{-1}$.

The comparison between the X-ray luminosities reported in the right and left panels of Fig. 6, as well as the relation between the ratio $l_{\max } / h_{\text {sink }}$ and the model parameters (Fig. 7), allow us to highlight the role of absorption from the stellar atmosphere on the observability of the post-shock zone in the X-ray band as a function of the accretion stream properties. Specifically, we find that:

- Low density $\left(n_{\text {acc }} \leq 10^{11} \mathrm{~cm}^{-3}\right)$ and high velocity $\left(u_{\text {acc }} \geq\right.$ $400 \mathrm{~km} \mathrm{~s}^{-1}$ ) streams form shocked slabs with X-ray emission poorly absorbed by the surrounding stellar chromosphere, the thickness of the post-shock zone being much larger than the sinking of the stream in the chromosphere $\left(l_{\max } \gg h_{\text {sink }}\right)$, 


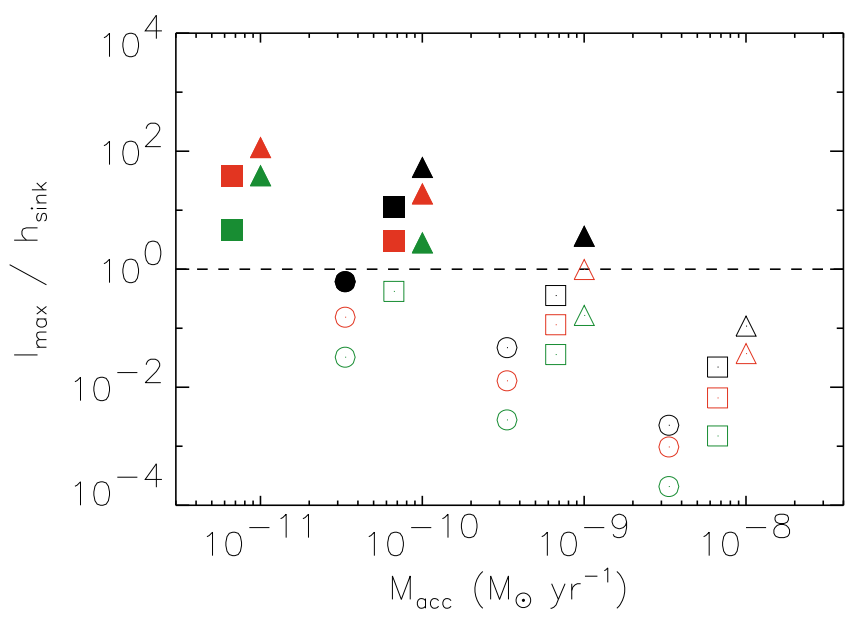

Fig. 7. The ratio $l_{\max } / h_{\text {sink }}$ as function of the mass accretion rate. Filled and empty symbols indicate if X-ray emission from the stream is observable or absorbed, respectively. Symbols and colors as in Fig. 4.

so that most of the emitting plasma is located in the shallower and low density portion of the chromosphere.

- High density $\left(n_{\text {acc }} \geq 10^{13} \mathrm{~cm}^{-3}\right)$ and low velocity $\left(u_{\text {acc }} \leq\right.$ $200 \mathrm{~km} \mathrm{~s}^{-1}$ ) accretion streams form shocked slabs with $\mathrm{X}$-ray emission strongly absorbed by the chromosphere, being in these cases $l_{\mathrm{ps}} \ll h_{\text {sink }}$.

- The effects of the absorption in the case of intermediate density $\left(n_{\text {acc }} \sim 10^{11}-10^{12} \mathrm{~cm}^{-3}\right)$ streams are less evident, especially for those cases with $l_{\max } \sim h_{\text {sink. }}$. Our results suggest that except for the stream with $u_{\mathrm{acc}}=600 \mathrm{~km} \mathrm{~s}^{-1}$ and metal abundance $\zeta=0.2$, all the streams with density $n_{\text {acc }} \approx 10^{12} \mathrm{~cm}^{-3}$ generate shocked slab whose X-ray emission is strongly absorbed (see Fig. 7). However, these intermediate cases probably require a more accurate analysis of the absorption effect that considers: a) the absorption dependence on the wavelength; b) a detailed model describing the absorption from the stellar atmosphere with a more smooth transition between the optically thin and the optically thick case and different angles of view; c) different metal abundances in the accretion stream and in the surrounding atmosphere.

It is worth to emphasize that if accretion streams are not uniform in density, the chromospheric absorption triggers a selection effect, absorbing preferentially the X-ray emission from high density plasma components. In addition, since the chromospheric absorption does not equally affect the different density tracers (e.g. O VII and Ne IX He-like triplets; see right panels in Fig. 6), we expect that, in general, the results of the use of these tracers may differ significantly (see also discussion at the end of Sect. 4).

\section{Discussion}

Observational data and 3D magnetospheric models of the stardisk interaction in young stars draw a very complex scenario (see Bouvier et al. 2007, for a review of the recent results), where circumstellar gas accretes onto the star through many streams observed from different angles of view and, likely, non-uniform in density. Our modeling focuses on the portion of the stream impacting on the chromosphere where a shock develops and assumes the stream with a constant mass accretion rate. It is worth to emphasize, however that the model results presented here can be combined together in a tool-box like fashion, if the plasma$\beta \ll 1$ ( assumption which is valid for many CTTSs as discussed in Sect. 2.2), allowing us to build up inhomogeneous streams as bundles of independent fibrils, each describable in terms of a different 1-D model (see discussion in Sect. 3.1). This method can be easily applied to investigate the X-ray emission expected from a 3D density structured accretion flow, adding together the contribution to the emission of the distribution of fibrils each with its own density. In the following, we apply this idea to very simplified cases. In the future the method could be applied to describe more complex configurations.

The hypothesis that the soft X-ray emission from CTTSs is mainly due to post-shock accreting plasma is supported by the evidence that the emission originates from high density plasma $\left(n_{\mathrm{e}}>10^{11} \mathrm{~cm}^{-3}\right)$. This evidence has been found in all (six) CTTSs with mass lower than $1.2 M_{\odot}$ observed to date by high resolution X-ray spectrographs. In addition, no signatures of high density plasma have been revealed in current X-ray observations of weak-line T Tauri stars (e.g. Kastner et al. 2004; Argiroffi et al. 2005) in which accretion is known to be absent.

However, although the soft X-ray excess in CTTSs can be interpreted as due to accretion shocks, some observational results are still not easily explained in terms of post-shock accreting plasma: a) if we assume to observe all the energy emitted by the post-shock zone, the accretion rates derived from X-ray data are generally about $1-2$ orders of magnitude lower than those derived from optical data (Schmitt et al. 2005; Günther et al. 2007; Argiroffi et al. 2009; Curran et al. 2010); b) plasma densities derived from the O VII triplet are generally lower than those derived from the Ne IX triplet (Brickhouse et al. 2010), although we expect the opposite result as the Ne IX triplet forms at higher temperatures than O VII where accretion shock models predict lower densities (e.g. Sacco et al. 2008); c) no signatures of high density plasma have been found in the high mass $\left(M>2 M_{\odot}\right)$ accreting objects, namely T Tau, AB Aur and HD 163296 (Güdel et al. 2007; Telleschi et al. 2007; Günther \& Schmitt 2009).

The above issues can be addressed in the light of the results discussed in Sect. 3. The comparison of the right and left panels of Fig. 6 highlights the importance of the absorption from the optically thick plasma of the chromosphere in the selection of post-shock plasma components that produce observable emission in the X-ray band. As a consequence, we found that the $\mathrm{X}$-ray luminosity of the post-shock zone as well as the mass accretion rate derived from the emerging X-ray emission strongly depend on absorption effects.

Our exploration of the model parameter space has shown that only a fraction of modeled streams predict post-shock plasma with measurable X-ray emission. Interestingly, in five out of six CTTSs (MP Mus, BP Tau, TW Hydrae, V4046 Sgr and RU Lupi) observed with high resolution X-ray spectrographs, the preshock densities derived from the He-like triplet $\left(10^{11} \lesssim n_{\text {acc }} \lesssim\right.$ $\left.10^{12} \mathrm{~cm}^{-3}\right)$, the escape velocities $\left(400 \lesssim u_{\text {acc }} \lesssim 600 \mathrm{~km} \mathrm{~s}^{-1}\right)$ and the metal abundances $(\zeta \leq 1)$ lie in the ranges of values that, according to the plots in Fig. 6 , are the most suitable for the observation of X-ray emission from the post-shock accreting plasma. The CTTS Hen 3-600 lies at the boundaries of our range of observability due to its low escape velocity $\left(u_{\mathrm{acc}} \sim 290 \mathrm{~km} \mathrm{~s}^{-1}\right)$ and the nearly solar metal abundances $(\zeta \sim 1.0)$.

Our results can help us to reconcile the (apparent) discrepancy found between the accretion rates measured from X-rays and optical data (Schmitt et al. 2005; Günther et al. 2007; Argiroffi et al. 2009; Curran et al. 2010). In fact, in the case of accretion streams with non-uniform mass density along the stream cross section (or in the case of multiple streams with 
different density simultaneously present on the star), we expect to observe X-rays preferentially from the low density portion of the post-shock plasma, the high density shocked plasma being strongly absorbed. For instance, as suggested by 3D MHD models of the star-disk system (Romanova et al. 2004), accretion streams may consist of a high density central region, surrounded by material at lower densities. Consequently, the shocked slab should be constituted by a dense core (rooted deeply in the chromosphere and with a short stand-off height) surrounded by less dense shocked material (located shallower in the chromosphere and with a large stand-off height). According to our results in Sect. 3, the X-ray emission from the dense core is expected to be largely absorbed by the stellar chromosphere and by the accretion stream itself, whereas X-rays emitted from the (less dense) shocked plasma at the boundary of the slab may suffer only a minor absorption by the chromosphere. All the shocked plasma contributes to the optical emission, whereas only a fraction of it contributes to the $\mathrm{X}$-ray emission. The result is that the accretion rates deduced from optical observations are expected to be larger than the rate values deduced from X-ray observations.

Under the assumption that the accretion streams are inhomogeneous and taking into account the chromospheric absorption, our model predicts that different He-like triplets, in general, measure different densities of the emitting plasma. In fact, since the effects of absorption increases with wavelength, Ne IX (13.45 $\AA$ ) emission is expected to be less absorbed than O VII (21.60 $\AA$ ) emission. Consequently, the high density plasma (i.e. the component suffering more absorption effects) should contribute more to Ne IX than to O VII emission. In the case of inhomogeneous streams, therefore the average density of the plasma leading to Ne IX emission is expected to be larger that that of plasma leading to O VII emission. This argument can be checked with a simple case of an accretion stream with velocity $u_{\text {acc }}=600 \mathrm{~km} \mathrm{~s}^{-1}$, metal abundance $\zeta=0.2$ and two components with the same cross section, one with density $n_{\text {acc }}=10^{11} \mathrm{~cm}^{-3}$ and the other with $n_{\text {acc }}=10^{12} \mathrm{~cm}^{-3}$. From Table 3, we obtain that the X-ray luminosities in the OVII resonance line due to the low and high density stream components $\operatorname{are} \log \left(L_{\mathrm{OVII}}\right)=28.85$ and $\log \left(L_{\text {OVII }}\right)=28.9 \mathrm{erg} \mathrm{s}^{-1}$, respectively, whereas the luminosities in the Ne IX resonance line are $\log \left(L_{\mathrm{NeIX}}\right)=28.53$ and $\log \left(L_{\mathrm{NeIX}}\right)=29.04 \mathrm{erg} \mathrm{s}^{-1}$. In other words, the denser stream contribute for $73 \%$ to the Ne IX emission, but only for $53 \%$ to the $\mathrm{O}$ VII emission. The average density measured with the Ne IX triplet is expected therefore to be larger than that measured with O VII triplet although Ne IX lines form at temperatures higher than O VII lines.

Finally, our results do not allow us to formulate a unique hypotheses to explain why soft X-ray emission from high density plasma have not been detected in high mass young accreting objects (e.g. T Tau, AB Aur and HD 163296; Güdel et al. 2007; Telleschi et al. 2007; Günther \& Schmitt 2009). In particular two hypotheses can be formulated: a) accretion streams in these objects are, in general, characterized by densities higher than $n_{\text {acc }}=10^{12} \mathrm{~cm}^{-3}$, so that the X-ray emission from shocked material is fully absorbed by the chromosphere; b) accretion streams are much less dense than streams of low-mass stars $\left(n_{\text {acc }}<10^{10} \mathrm{~cm}^{-3}\right)$, so that the resulting X-ray emission from the shocked material cannot be distinguished from the coronal emission. Further analysis of optical and X-ray observations of high mass young accreting objects is needed to assess if the lack of X-ray emission from high density plasma is a general feature of these objects and if some hints on the density of accretion streams can be obtained.

\section{Conclusions}

We performed an intensive simulation campaign, using the 1-D hydrodynamic model introduced in Paper I, exploring the model parameter space which is representative of almost all low-mass CTTSs observed to date in the X-ray band. The model describes the impact of an accretion stream onto the chromosphere of a CTTS under the hypothesis that $\beta \ll 1$. Our aims include: (1) to investigate the physical properties of shocked slab resulting from the stream impact as a function of the density, velocity and metal abundance of the accretion stream and (2) to investigate the observability of the post-shock plasma in the X-ray band, taking into account the absorption from the optically thick plasma of the surrounding chromosphere. Our main results can be summarized as follows:

1. The post-shock zone resulting from the impact of the accretion stream onto the stellar surface is characterized by temperatures ranging from 0.5 to $5 \mathrm{MK}$ (depending on the stream velocity) and by stand-off heights of the postshock zone spanning six orders of magnitude from $\approx 10^{4}$ to $\approx 10^{10} \mathrm{~cm}$ (mainly depending on the stream density).

2. In all the case the post-shock zone oscillates quasiperiodically. Oscillations are composed by a heating phase during which the accretion shock builds up a slab of hot plasma and a cooling phase during which the post-shock zone cools down under the effect of thermal instabilities at its base. The oscillation period ranges from $\approx 3 \times 10^{-2}$ to $\approx 4 \times 10^{3} \mathrm{~s}$ within the space of physical parameters. However, these oscillations are very difficult to observe, because the accretion stream is, most likely, inhomogeneous and clumped (i.e. with variable accretion rate) and, in the case of $\beta \ll 1$, constituted by several different fibrils with different instability periods and random phases, leading to no evident periodic variations of the X-ray emission ${ }^{1}$.

3. The effect of the absorption from the local chromosphere on the X-ray emission from the post-shock zone strongly depends on the accretion stream properties. The stream density is a key parameter to make observable in the X-ray band the post-shock material: the higher the stream density, the thinner and more deep rooted in the chromosphere the shocked slab, and the larger the absorption of X-ray emission from the slab. We found that, in general, high density accretion streams $\left(n_{\text {acc }}>10^{12} \mathrm{~cm}^{-3}\right)$ produce shocked slab which are strongly absorbed. Shocked plasma from high velocity streams is more easily observable than that from low velocity ones, the stand-off heights of the shocked slab increasing rapidly with the stream velocity.

4. Our results suggest that the discrepancy between the mass accretion rates derived from optical and X-ray data as well as the different densities measured, in general, from the $\mathrm{O}$ VII and Ne IX line triplets diagnostics can be explained if accretion streams are inhomogeneous (and/or multiple streams with different densities are present simultaneously). In fact, only the light component of the post-shock plasma is preferentially observed in the X-ray band, leading to an underestimation of the mass accretion rate with respect to the rate value deduced from optical observations. Also, the absorption in O VII lines is larger than in Ne IX lines and, therefore, the weight of dense plasma is expected to be larger in Ne IX than in $\mathrm{O}$ VII.

Acknowledgements. Research on X-rays from young stars by G.S. is supported by NASA/Goddard XMM-Newton Guest Observer Facility grants

${ }^{1}$ In the case of $\beta \geq 1$, see discussion in Orlando et al. (2010). 
NNX09AT15G and NNX09AC11G and NASA Astrophysics Data Analysis program grant NNX09AC96G to RIT. This work was supported in part by the Italian Ministry of University and Research (MIUR) and by Istituto Nazionale di Astrofisica (INAF). We acknowledge support through the EU Marie Curie Transfer of Knowledge program PHOENIX under contract No. MTKD-CT2005-029768. The software used in this work was in part developed by the DOEsupported ASC / Alliance Center for Astrophysical Thermonuclear Flashes at the University of Chicago. The simulations have been executed at the HPC facility (SCAN) of the INAF - Osservatorio Astronomico di Palermo.

\section{References}

Anders, E., \& Grevesse, N. 1989, Geochim. Cosmochim. Acta, 53, 197 Ardila, D. R., Basri, G., Walter, F. M., Valenti, J. A., \& Johns-Krull, C. M. 2002, ApJ, 566, 1100

Argiroffi, C., Maggio, A., Peres, G., Stelzer, B., \& Neuhäuser, R. 2005, A\&A, 439, 1149

Argiroffi, C., Maggio, A., \& Peres, G. 2007, A\&A, 465, L5

Argiroffi, C., Maggio, A., Peres, G., et al. 2009, A\&A, 507, 939

Bary, J. S., Matt, S. P., Skrutskie, M. F., et al. 2008, ApJ, 687, 376

Borkowski, K. J., Shull, J. M., \& McKee, C. F. 1989, ApJ, 336, 979

Bouvier, J., Alencar, S. H. P., Harries, T. J., Johns-Krull, C. M., \& Romanova, M. M. 2007, Protostars and Planets V, 479

Brickhouse, N. S., Cranmer, S. R., Dupree, A. K., Luna, G. J. M., \& Wolk, S. 2010, ApJ, 710, 1835

Brown, J. C. 1973, Sol. Phys., 29, 421

Calvet, N., \& Gullbring, E. 1998, ApJ, 509, 802

Colella, P., \& Woodward, P. R. 1984, J. Comput. Phys., 54, 174

Cowie, L. L., \& McKee, C. F. 1977, ApJ, 211, 135

Curran, R. L., Argiroffi, C., Sacco, G. G., et al. 2010, A\&A, submitted

Dalton, W. W., \& Balbus, S. A. 1993, ApJ, 404, 625

Dopita, M. A., \& Sutherland, R. S. 1996, ApJS, 102, 161

Drake, J. J. 2005, in 13th Cambridge Workshop on Cool Stars, Stellar Systems and the Sun, ed. F. Favata, G. A. J. Hussain, \& B. Battrick, ESA SP, 560, 519

Drake, J. J., Ratzlaff, P. W., Laming, J. M., \& Raymond, J. 2009, ApJ, 703, 1224

Edwards, S., Hartigan, P., Ghandour, L., \& Andrulis, C. 1994, AJ, 108, 1056

Fryxell, B., Olson, K., Ricker, P., et al. 2000, ApJS, 131, 273

Güdel, M., Skinner, S. L., Mel’Nikov, S. Y., et al. 2007, A\&A, 468, 529

Gullbring, E. 1994, A\&A, 287, 131

Gullbring, E., Calvet, N., Muzerolle, J., \& Hartmann, L. 2000, ApJ, 544, 927

Günther, H. M., \& Schmitt, J. H. M. M. 2009, A\&A, 494, 1041
Günther, H. M., Liefke, C., Schmitt, J. H. M. M., Robrade, J., \& Ness, J.-U. 2006, A\&A, 459, L29

Günther, H. M., Schmitt, J. H. M. M., Robrade, J., \& Liefke, C. 2007, A\&A, 466, 1111

Herczeg, G. J., Walter, F. M., Linsky, J. L., et al. 2005, AJ, 129, 2777

Huenemoerder, D. P., Kastner, J. H., Testa, P., Schulz, N. S., \& Weintraub, D. A. 2007, ApJ, 671, 592

Johns-Krull, C. M. 2007, ApJ, 664, 975

Kashyap, V., \& Drake, J. J. 2000, Bull. Astron. Soc. India, 28, 475

Kastner, J. H., Huenemoerder, D. P., Schulz, N. S., Canizares, C. R., \& Weintraub, D. A. 2002, ApJ, 567, 434

Kastner, J. H., Huenemoerder, D. P., Schulz, N. S., et al. 2004, ApJ, 605, L49

Koenigl, A. 1991, ApJ, 370, L39

Koldoba, A. V., Ustyugova, G. V., Romanova, M. M., \& Lovelace, R. V. E. 2008, MNRAS, 388, 357

Lamzin, S. A. 1998, Astron. Rep., 42, 322

MacNeice, P., Olson, K. M., Mobarry, C., de Fainchtein, R., \& Packer, C. 2000, Comput. Phys. Commun., 126, 330

Martin, S. C. 1996, ApJ, 470, 537

Mignone, A. 2005, ApJ, 626, 373

Orlando, S., Peres, G., Reale, F., et al. 2005, A\&A, 444, 505

Orlando, S., Sacco, G. G., Argiroffi, C., et al. 2010, A\&A, 510, A71

Peres, G., Serio, S., Vaiana, G. S., \& Rosner, R. 1982, ApJ, 252, 791

Robrade, J., \& Schmitt, J. H. M. M. 2007, A\&A, 473, 229

Romanova, M. M., Ustyugova, G. V., Koldoba, A. V., \& Lovelace, R. V. E. 2004, ApJ, 610, 920

Sacco, G. G., Argiroffi, C., Orlando, S., et al. 2008, A\&A, 491, L17

Safier, P. N. 1998, ApJ, 494, 336

Schmitt, J. H. M. M., Robrade, J., Ness, J.-U., Favata, F., \& Stelzer, B. 2005, A\&A, 432, L35

Smith, R. K., Brickhouse, N. S., Liedahl, D. A., \& Raymond, J. C. 2001, ApJ, 556, L91

Spitzer, L. 1962, Physics of Fully Ionized Gases (New York: Wiley)

Sutherland, R. S., \& Dopita, M. A. 1993, ApJS, 88, 253

Sutherland, R. S., Bicknell, G. V., \& Dopita, M. A. 2003a, ApJ, 591, 238

Sutherland, R. S., Bisset, D. K., \& Bicknell, G. V. 2003b, ApJS, 147, 187

Telleschi, A., Güdel, M., Briggs, K. R., et al. 2007, A\&A, 468, 541

Testa, P., Drake, J. J., \& Peres, G. 2004, ApJ, 617, 508

Vernazza, J. E., Avrett, E. H., \& Loeser, R. 1973, ApJ, 184, 605

Zel'Dovich, Y. B., \& Raizer, Y. P. 1967, Physics of shock waves and hightemperature hydrodynamic phenomena, ed. W. D. Hayes, \& R. F. Probstein (New York: Academic Press) 